\title{
Time-Varying Feedback Control for Nonholonomic Mobile Robots Forming Group Formations
}

\author{
Hiroaki Yamaguchi and Joel W. Burdick \\ Department of Mechanical Engineering \\ Division of Engineering and Applied Science \\ CALIFORNIA INSTITUTE OF TECHNOLOGY \\ Mail Code 104-44, Pasadena, CA 91125, USA \\ yamaguch@robby.caltech.edu, jwb@robby.caltech.edu
}

\begin{abstract}
This paper presents a novel approach of control for multiple nonholonomic wheeled mobile robots of the Hilare-type to form group formations. To control the formation, each robot has its own coordinate system and it controls its relative positions to its neighboring robots. Particularly, it has a vector called "a formation vector," and the formation is controllable by the vectors. Since the robots have nonholonomic constraints, it is not possible for them to directly move in omni-directions, which means that such nonholonomic vehicles cannot be asymptotically stabilized by smooth static-state feedback control laws. We introduce a smooth time-varying feedback control law whose asymptotic stability is guaranteed in a mathematical framework, averaging theory. The validity of this law is verified by computer simulations.
\end{abstract}

\section{Introduction}

Feedback control is always one of the most challenging subjects in engineering and it is closely related with mathematics especially in its proofs of stability. This paper introduces and describes a paradigm, control of multiple nonholonomic wheeled mobile robots to form group formations, and proposes a smooth time-varying feedback control law whose asymptotic stability is guaranteed in a mathematical framework, averaging theory.

As Brockett's theorem [1] suggests, controllable systems without drift (including unicycle-type vehicles, car-like vehicles and vehicles with n-trailers) cannot be asymptotically stabilized by smooth static-state feedback control laws. In recent years, feedback control laws for such mechanical systems have been intensively studied [2]-[9]. Samson [2] initiated to examine smooth time-varying feedback control laws and presented a control method for an unicycle-type vehicle, which is kinematically equivalent to a Hilare-type mobile robot. Motivated by the potentialities of such feedback control laws, Coron [3] has proven that smooth time-varying feedback control laws can asymptotically stabilize controllable systems without drift. This result showed the existence of the control laws and then Pomet [4] presented an explicit design procedure for them based on the Lyapunov second method. Teel et al. [5] presented another smooth time-varying feedback control law to asymptotically stabilize mechanical systems in power form. Samson [6] presented skew-symmetric chain form and showed that mechanical systems in this form can be also asymptotically stabilized by a smooth time-varying feedback control law. As alternatives, piecewise, discontinuous and nonsmooth feedback control laws have been explored. Bloch et al. [7] presented a piecewise analytic feedback control law to asymptotically stabilize a class of nonholonomic mechanical systems. Canudas de Wit and Sørdalen [8] presented a discontinuous feedback control law to exponentially stabilize unicycle-type vehicles in three dimensional chained form. Sørdalen and Egeland [9] presented a nonsmooth time-varying feedback control law to exponentially stabilize all mechanical systems in chained form. These control laws are supposed to control a single mechanical system.

In this paper, we present a smooth time-varying feedback control law for multiple Hilare-type mobile robots to form group formations. Unlike other feedback control laws which have particular form (e.g., chained, power and skew-symmetric chain form [5][6][8][9]) and whose asymptotic stability is guaranteed based on the Lyapunov second method [2][4][6], this feedback control law does not require any particular form to describe kinematics of mobile robots and its asymptotic stability is guaranteed in averaging theory. To prove its asymptotic stability, we apply a theorem in averaging theory given by Eckhaus and Sanchez-Palencia [10]. We specifically deal with a matrix whose components are averaged over time by integration and whose eigenvalue distribution explicitly describes stability of our control method. Since the components of 
this matrix are integral functions, its eigenvalue distribution is analytically shown by Hölder's inequality in functional analysis [11]. Hence, stability analysis of our control method is given in a mathematical framework, averaging theory, and it is also technically related with functional analysis.

\section{Model of Mobile Robot and Robot Group}

\subsection{Model of Mobile Robots}

We consider ( $\mathrm{n}$ ) mobile robots on a plane. Each robot is a Hilare-type mobile robot that has two wheels driven independently. We label a robot $R_{1}$, but this label is not intrinsic to this method. Each robot does not need to know its own label and other robots' labels. To form a group formation, each robot senses its relative positions to its neighboring robots in its own coordinate system, $\Sigma_{\mathrm{i}}$. Let us call the robots sensed by $R_{\mathrm{i}}$ "the neighboring robots of $R_{\mathrm{i}}$." To express the positions of all the robots in a common coordinate system, we define a static coordinate system, $\Sigma_{0}$. To express the orientation of $R_{1}$, we define a coordinate system, $\Sigma_{i}^{0}$, whose origin is common to that of $\Sigma_{0}$ and whose orientation is common to that of $\Sigma_{i}$, (see Figure 1). However, each robot knows only $\Sigma_{\mathrm{i}}$ and it does not need to know $\Sigma_{0}$ and $\Sigma_{i}^{0}$.

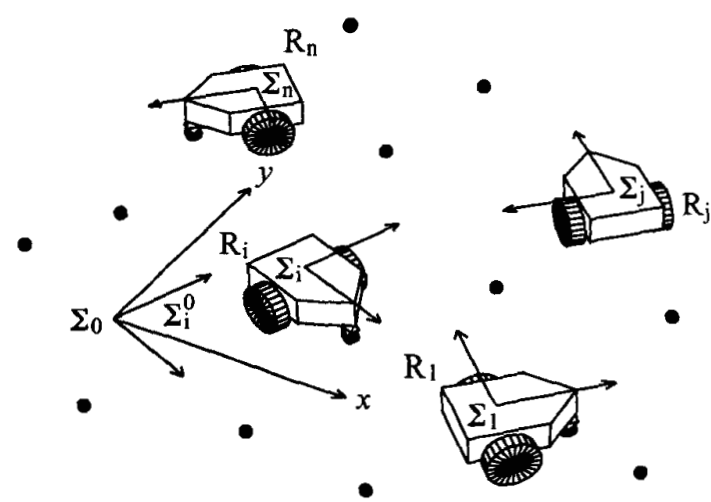

Figure 1 Multiple Nonholonomic Mobile Robots

\subsection{Model of Robot Group}

Each robot senses its relative positions to its neighboring robots. In this case, we can consider possible three pairs of robots: both sensing; only one sensing; and neither sensing. An arrow, (see Figure 3 in Section 5), means that a robot at the start of the arrow is sensing another robot at the end of the arrow. We consider only cases of formation control for open chain groups. Using graph theory terminology, we can see that an open chain group has a strongly connected configuration. We assume that the robot group has organized this configuration before it starts to form a group formation, and the configuration is always static.

\section{Robot Control}

\subsection{Desired Velocity}

Since, as we have described, (n) mobile robots form an open chain group, each mobile robot senses one or two mobile robots. We denote the set of robots sensed by $R_{i}$ with $\mathrm{L}_{\mathrm{i}}$. To form a desired formation, each robot tries to keep its desired relative positions to its neighboring robots. Specifically, when its relative positions are different from its desired ones, it calculates its desired velocity and it tries to move at this velocity until it has the desired relative positions. We propose to determine the desired velocity as Eq. (1):

$\tilde{\boldsymbol{v}}_{i}^{i}=\left(\begin{array}{c}\tilde{v}_{x i}^{i} \\ \tilde{v}_{y i}^{i}\end{array}\right)=\sum_{j \in \mathrm{L}_{i}} \tau_{i j}\left(\begin{array}{c}x_{j}^{i} \\ y_{j}^{i}\end{array}\right)+\left(\begin{array}{c}d_{x i}^{i} \\ d_{y i}^{i}\end{array}\right)$,

where $\tilde{v}_{i}^{i}$ is the desired velocity of $\mathrm{R}_{\mathrm{i}}$ in $\Sigma_{\mathrm{i}} ;{ }^{t}\left(x_{j}^{i}, y_{j}^{i}\right)$ is the position of $R_{j}$ in $\Sigma_{i}$, i.e., the relative position of $R_{j}$ to $R_{i}$ in $\Sigma_{i}{ }^{t}\left(d_{x i}^{i}, d_{y i}^{i}\right)$ is the formation vector of $R_{i}$ in $\Sigma_{i}$; and $\tau_{i j}$ is the attraction coefficient of $R_{i}$ to $R_{j}$. Physically, the term, $\tau_{i j}{ }^{t}\left(x_{j}^{i}, y_{j}^{i}\right)$, means that $R_{\mathrm{i}}$ is attracted to $\mathrm{R}_{\mathrm{j}}$. The formation vector, ${ }^{t}\left(d_{x i}^{i}, d_{y i}^{i}\right)$, means that $R_{\mathrm{i}}$ is pulled in the direction of this vector. This desired velocity is expressed in the static coordinate system, $\Sigma_{0}$, as Eq.(2):

$$
\begin{aligned}
\tilde{\boldsymbol{v}}_{\boldsymbol{i}} & =\left(\begin{array}{c}
\tilde{v}_{x i} \\
\tilde{v}_{y i}
\end{array}\right)=\rho_{i} \tilde{\boldsymbol{v}}_{\boldsymbol{i}}^{i}=\sum_{j \in \mathrm{L}_{\mathrm{i}}} \tau_{i j} \rho_{i}\left(\begin{array}{c}
x_{j}^{i} \\
y_{j}^{i}
\end{array}\right)+\rho_{i}\left(\begin{array}{c}
d_{x i}^{i} \\
d_{y i}^{i}
\end{array}\right) \\
& =\sum_{j \in \mathrm{L}_{\mathbf{i}}} \tau_{i j}\left\{\left(\begin{array}{c}
x_{j} \\
y_{j}
\end{array}\right)-\left(\begin{array}{c}
x_{i} \\
y_{i}
\end{array}\right)\right\}+\left(\begin{array}{c}
d_{x i} \\
d_{y i}
\end{array}\right),
\end{aligned}
$$

where $\rho_{i}$ is the transformation matrix from $\Sigma_{\mathrm{i}}^{0}$ to $\Sigma_{0}$; ${ }^{t}\left(x_{j}, y_{j}\right)$ is the position of $\mathrm{R}_{\mathrm{j}}$ in $\Sigma_{0} ;{ }^{t}\left(x_{i}, y_{i}\right)$ is the position of $\mathrm{R}_{\mathrm{i}}$ in $\Sigma_{0}$; and ${ }^{t}\left(d_{x i}, d_{y i}\right)$, is the formation vector expressed in $\Sigma_{0}$.

\subsection{Desired System}

Let us consider a case where the robot is a holonomic robot and it can move at its desired velocity. Although, in reality, the robot is a nonholonomic mobile robot and it cannot move sideways, we dare to assume this case here and let us discuss stability of the robot controlled by Eq.(1) in this subsection. We call this robotic system "a desired system." Since the nonholonomic robot in our control method tries to move at its desired velocity, at least this desired system should be stable. To analyze stability of the desired system, we rewrite Eq.(2) as Eq.(3):

$\left(\begin{array}{l}\bar{v}_{x} \\ \bar{v}_{\boldsymbol{y}}\end{array}\right)=\left(\begin{array}{cc}B & 0 \\ 0 & B\end{array}\right)\left(\begin{array}{l}x \\ y\end{array}\right)+\left(\begin{array}{l}d_{x} \\ d_{y}\end{array}\right)$, 
where $\tilde{v}_{\boldsymbol{x}}={ }^{t}\left(\tilde{v}_{x 1}, \tilde{v}_{x 2}, \cdots, \tilde{v}_{x n}\right) ; \tilde{\boldsymbol{v}}_{\boldsymbol{y}}={ }^{t}\left(\tilde{v}_{y 1}, \tilde{v}_{y 2}, \cdots, \tilde{v}_{y n}\right)$; $\boldsymbol{x}={ }^{t}\left(x_{1}, x_{2}, \cdots, x_{n}\right) ; \boldsymbol{y}={ }^{t}\left(y_{1}, y_{2}, \cdots, y_{n}\right) ; \quad \boldsymbol{d}_{\boldsymbol{x}}=$ ${ }^{t}\left(d_{x 1}, d_{x 2}, \cdots, d_{x n}\right)$; and $\boldsymbol{d}_{\boldsymbol{y}}={ }^{t}\left(d_{y 1}, d_{y 2}, \cdots, d_{y n}\right)$. The components of $B \in \mathbf{R}^{n \times n}$ are given as: $b_{i j, i \neq j}=\tau_{i j \in \mathrm{L}_{\mathrm{i}}}>0$, $b_{i j, i \neq j}=\tau_{i j \notin \mathrm{L}_{\mathrm{i}}}=0$, and $b_{i i}=-\Sigma_{j=1, j \neq i}^{n} b_{i j}<0$. Since all the diagonal components of $B$ are negative and all the non-diagonal components are not negative, $B$ is a compartment matrix. As the summation of all the column vectors of $B$ is zero, one of the eigenvalues of $B$ is zero. The eigenvector of this zero-eigenvalue is parallel to ${ }^{t}(1,1, \cdots, 1) \in \mathbf{R}^{n}$. This eigenvector physically means that the formation is independent of the position of the group in $\Sigma_{0}$. Using Geršgorin's and Perron-Frobenius theorems [12], we can prove that all the eigenvalues of $B$ are asymptotically stable except only one zero-eigenvalue.

All the robots in this desired system move at their desired velocities, i.e., $\dot{\boldsymbol{x}}=\tilde{\boldsymbol{v}}_{\boldsymbol{x}}$ and $\dot{\boldsymbol{y}}=\tilde{\boldsymbol{v}}_{\boldsymbol{y}}$ hold, so that Eq.(3) is rewritten as Eq.(4):

$$
\left(\begin{array}{c}
\dot{\boldsymbol{x}} \\
\dot{\boldsymbol{y}}
\end{array}\right)=\left(\begin{array}{cc}
B & 0 \\
0 & B
\end{array}\right)\left(\begin{array}{l}
\boldsymbol{x} \\
\boldsymbol{y}
\end{array}\right)+\left(\begin{array}{l}
\boldsymbol{d}_{\boldsymbol{x}} \\
\boldsymbol{d}_{\boldsymbol{y}}
\end{array}\right)
$$

As we have described above, $B$ has a zero-eigenvalue that is stable but not asymptotically stable. Hence, this system has a possibility of being unstable. Let us consider a vector space, $S=\left\{\boldsymbol{\zeta} ; \boldsymbol{\zeta} \in V^{n}, \boldsymbol{\zeta}=B \boldsymbol{\xi}\left(\boldsymbol{\xi} \in V^{n}\right)\right\}$, where $V^{n}$ is a (n)-dimensional vector space. Since $B$ has a zeroeigenvalue, $\operatorname{dim}(S)=n-1$. When $\boldsymbol{d}_{\boldsymbol{x}}, \boldsymbol{d}_{\boldsymbol{y}} \in S$, this desired system is stable, otherwise this system is unstable, e.g., all the robots drift at the same velocity. We can intuitively interpret such an unstable condition as follows. As we have described in Subsection 3.1, $\mathrm{R}_{\mathrm{i}}$ is attracted to its neighboring robots and it is pulled in the direction of the formation vector, ${ }^{t}\left(d_{x i}^{i}, d_{y i}^{i}\right)$. When these pulling forces balance, the robot group is at rest, otherwise the robot group drifts, keeping a group formation. Therefore, we have to design $d_{x}$ and $d_{y}$, satisfying this condition: $d_{x}, d_{y} \in S$.

\subsection{Control Inputs for Robot Wheels}

A Hilare-type mobile robot has two wheels that are driven independently. Rotating these wheels, this mobile robot controls its position and orientation. In particular, it is possible for this type of robot to change its orientation without moving in any direction. Bulldozers and tanks have this physical feature and these types of vehicles are more maneuverable than automobiles. We denote the position of $\mathrm{R}_{\mathrm{i}}$ with ${ }^{t}\left(x_{i}, y_{i}\right)$ and its orientation with $\theta_{i}$ in the static coordinate system, $\Sigma_{0}$. We also denote the rotation angles of its two wheels with $\phi_{i 1}$ and $\phi_{i 2}$, respectively, and the radius of the wheel with $R$ and the width between the wheels with $W$, (see Figure 2). Its moving velocity and its angular velocity of its orientation are given as Eq.(5):

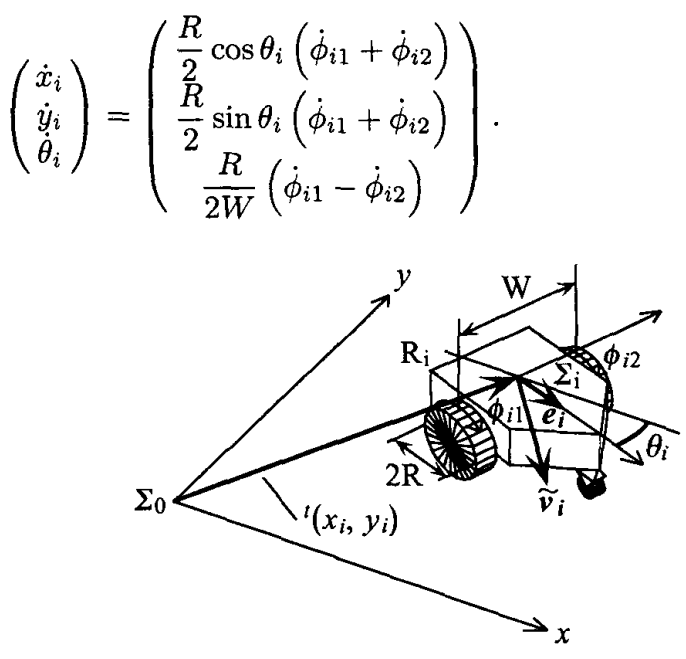

Figure 2 A Hilare-type Mobile Robot

We define $e_{i}$ in Eq.(6) that is an unit vector and that is on an axis of $\Sigma_{\mathrm{i}}$. The axis specifies the orientation of $\mathrm{R}_{\mathrm{i}}$, i.e., $\theta_{i}$ in the static coordinate system, $\Sigma_{0}$. We also define $v_{i}$ in Eq.(7) that is the velocity of $\mathrm{R}_{\mathrm{i}}$. These vectors, $\boldsymbol{e}_{\boldsymbol{i}}$ and $v_{i}$, are expressed in the static coordinate system, $\Sigma_{0}$.

$e_{i}={ }^{t}\left(\cos \theta_{i}, \sin \theta_{i}\right)$

$v_{i}={ }^{t}\left(\dot{x}_{i}, \dot{y}_{i}\right)=\frac{R}{2}\left(\dot{\phi}_{i 1}+\dot{\phi}_{i 2}\right) e_{i}$

As Eq.(7) shows, the robot can move only in the direction of the vector, $e_{i}$, and it cannot move in its sideways direction. This means that the robot cannot move at the desired velocity, $\tilde{\boldsymbol{v}}_{\boldsymbol{i}}$, as long as $\boldsymbol{e}_{\boldsymbol{i}}$ is not parallel to $\tilde{\boldsymbol{v}}_{\boldsymbol{i}}$. For that reason, we take a projection of $\tilde{\boldsymbol{v}}_{\boldsymbol{i}}$ onto the axis of $\Sigma_{\mathrm{i}}$ specifying $\theta_{i}$ in $\Sigma_{\mathbf{0}}$ as $\boldsymbol{e}_{\boldsymbol{i}} \tilde{\boldsymbol{v}}_{\boldsymbol{i}}$, and we determine the robot velocity, $v_{i}$, in order for it to be the same as the projection, as shown in Eq.(8):

$e_{i} v_{i}=e_{i} \tilde{v}_{i}$

From Eqs. $(7,8)$, the summation of the angular velocities of the two wheels is determined as Eq.(9):

$\dot{\phi}_{i 1}+\dot{\phi}_{i 2}=\frac{2}{R} e_{i} \tilde{v}_{i}$

Since the vector, $\tilde{v}_{i}$, linearly depends on the relative positions of $R_{i}$ to its neighboring robots, the right hand side of Eq.(9) is state feedback. When the robot does not change its orientation, $e_{i}$ is static and this feedback is static feedback. On the other hand, when the robot changes its orientation dependently on time, this feedback is time-varying feedback. Unless the robot changes its moving direction, the desired velocity, $\tilde{\boldsymbol{v}}_{\boldsymbol{i}}$, can be perpendicular to $\boldsymbol{e}_{\boldsymbol{i}}$. In the case where $\tilde{\boldsymbol{v}}_{\boldsymbol{i}} \perp \boldsymbol{e}_{\boldsymbol{i}}$, the projection of 
$\tilde{v}_{i}$ onto the axis of $\Sigma_{i}$, which specifies $\theta_{i}$ in $\Sigma_{0}$, is zero and the robot stops without forming a desired formation. To avoid this case, we propose that each robot changes its orientation according to time. Specifically, we design the orientation to be a function of time as $\theta_{i}=\theta_{i}(t)$. This means that we use time-varying feedback. Once this function is given and then the difference of the angular velocities between the wheels is determined as Eq.(10):

$\dot{\phi}_{i 1}-\dot{\phi}_{i 2}=\frac{2 W}{R} \dot{\theta}_{i}(t)$.

From Eqs. $(9,10)$, the angular velocities of the wheels are uniquely determined as Eqs. $(11,12)$ :

$\dot{\phi}_{i 1}=\left(e_{i} \tilde{v}_{i}+W \dot{\theta}_{i}(t)\right) / R$

$\dot{\phi}_{i 2}=\left(e_{i} \tilde{v}_{i}-W \dot{\theta}_{i}(t)\right) / R$.

An inner product, $\boldsymbol{e}_{\boldsymbol{i}} \tilde{\boldsymbol{v}}_{\boldsymbol{i}}$, in Eqs. $(11,12)$ can be rewritten as Eq.(13):

$e_{i} \tilde{v}_{i}=e_{i}^{i} \tilde{v}_{i}^{i}$

where $e_{i}^{i}$ and $\tilde{v}_{i}^{i}$ are vectors expressed in the coordinate system, $\Sigma_{\mathrm{i}}$. This means that $\mathrm{R}_{\mathrm{i}}$ can calculate this inner product without referring to the static coordinate system, $\Sigma_{0}$. Therefore, $R_{1}$ can determine its angular velocities of its wheels, $\dot{\phi}_{i 1}$ and $\dot{\phi}_{i 2}$, in $\Sigma_{\mathrm{i}}$ independently.

Consequently, the velocity of $R_{i}$ is given as Eq.(14):

$$
\begin{aligned}
& \left(\begin{array}{c}
\dot{x}_{i} \\
\dot{y}_{i}
\end{array}\right)=M_{i}\left(\begin{array}{c}
\tilde{v}_{x i} \\
\tilde{v}_{y i}
\end{array}\right) \\
& =M_{i}\left[\sum_{j \in \mathrm{L}_{i}} \tau_{i j}\left\{\left(\begin{array}{c}
x_{j} \\
y_{j}
\end{array}\right)-\left(\begin{array}{c}
x_{i} \\
y_{i}
\end{array}\right)\right\}+\left(\begin{array}{c}
d_{x i} \\
d_{y i}
\end{array}\right)\right], \\
& M_{i}=\left(\begin{array}{cc}
\cos ^{2} \theta_{i}(t) & \cos \theta_{i}(t) \sin \theta_{i}(t) \\
\sin \theta_{i}(t) \cos \theta_{i}(t) & \sin ^{2} \theta_{i}(t)
\end{array}\right) .
\end{aligned}
$$

As shown above, $M_{i}$ is a symmetrical matrix and its eigenvalues are real and its eigenvectors, which are perpendicular to each other, span a (2)-dimensional space. We shall regard the terms in the brackets of Eq.(14) as a vector field and we shall express it as a linear combination of the eigenvectors of $M_{i}$ as:

$\sum_{j \in \mathbf{L}_{i}} \tau_{i j}\left\{\left(\begin{array}{l}x_{j} \\ y_{j}\end{array}\right)-\left(\begin{array}{l}x_{i} \\ y_{i}\end{array}\right)\right\}+\left(\begin{array}{l}d_{x i} \\ d_{y i}\end{array}\right)=a_{i 1} w_{i \mathbf{1}}+a_{i 2} w_{i \mathbf{2}}$

where $\boldsymbol{w}_{i 1}$ and $\boldsymbol{w}_{i 2}$ are the eigenvectors of $M_{i}$. Then, Eq.(14) is rewritten as:

$$
\left(\begin{array}{c}
\dot{x}_{i} \\
\dot{y}_{i}
\end{array}\right)=M_{i}\left(a_{i 1} \boldsymbol{w}_{i \mathbf{1}}+a_{i 2} \boldsymbol{w}_{i \mathbf{2}}\right)=a_{i 1} \lambda_{i 1} \boldsymbol{w}_{i \mathbf{1}}+a_{i 2} \lambda_{i 2} \boldsymbol{w}_{i \mathbf{2}},
$$

where $\lambda_{i 1}$ and $\lambda_{i 2}$ are the eigenvalues of $M_{i}$. We can see that the right hand side of the above equation is a linear transformation of the vector field, $a_{i 1} w_{i 1}+a_{i 2} w_{i 2}$, by the matrix, $M_{i}$. The parameter, $a_{i 1}$, expresses the direction and the magnitude of a flow along $\boldsymbol{w}_{\boldsymbol{i 1}}$ in this vector field. Since the trace of this matrix is positive and its determinant is zero independently of $\theta_{i}(t)$, i.e., independently of the rotation of the robot, the matrix has a positive eigenvalue and a zero-eigenvalue. This zero-eigenvalue makes an equilibrium region (that is a line and that includes an equilibrium point of $\tilde{\boldsymbol{v}}_{\boldsymbol{i}}, \tilde{\boldsymbol{v}}_{\boldsymbol{i}}=0$ ), e.g., if $\lambda_{i 1}=0, \lambda_{i 1}$ makes the vector field, $a_{i 1} \boldsymbol{w}_{i \mathbf{1}}+a_{i 2} \boldsymbol{w}_{i \mathbf{2}}$, lose a flow along $\boldsymbol{w}_{\boldsymbol{i 1}}$ and it makes the equilibrium region (line) that is parallel to $w_{i 1}$ in this linear transformation, while $\lambda_{i 2}$, which is positive, does not change the direction of a flow along $\boldsymbol{w}_{\boldsymbol{i} \mathbf{2}}$ but changes the magnitude of the flow. Hence, unless the robot rotates, it is trapped by the equilibrium region where $\tilde{v}_{i} \perp \boldsymbol{e}_{i}$. Of course, we cannot conclude from Eq. (14) that the rotating robots can avoid to be trapped. We can just conclude from Eq.(14) that the robots stop without forming a desired formation at least when they do not rotate, because $M_{i}$ is not a full-rank matrix. However, we strongly emphasize here that, in the averaged system of Eq.(14) (which we discuss below), $M_{i}$ turns into a full-rank matrix whose eigenvalues are positive when the robots rotate and we can see that they form a desired formation.

\subsection{Averaged System}

We introduce an analysis method based on averaging theory, to examine stability of this control system. As we have described in Subsection 3.3, we design the orientation of the robot to be a function of time. Not in all cases but in some cases, we can examine stability of dynamical systems by analyzing their averaged systems that are independent of time. Obviously, analysis of time-independent dynamical systems is rather easier than analysis of time-dependent dynamical systems. Especially, stability of this control system can be examined by averaging theory. Moreover, its averaged system reflects its desired system whose stability has been already discussed in Subsection 3.2.

The averaged system of Eq.(14) is given as Eq.(15):

$$
\begin{aligned}
& \left(\begin{array}{c}
\dot{X}_{i} \\
\dot{Y}_{i}
\end{array}\right)=\mathcal{M}_{i}\left(\begin{array}{c}
\tilde{V}_{x i} \\
\tilde{V}_{y i}
\end{array}\right) \\
& =\mathcal{M}_{i}\left[\sum_{j \in \mathrm{L}_{i}} \tau_{i j}\left\{\left(\begin{array}{c}
X_{j} \\
Y_{j}
\end{array}\right)-\left(\begin{array}{c}
X_{i} \\
Y_{i}
\end{array}\right)\right\}+\left(\begin{array}{c}
d_{x i} \\
d_{y i}
\end{array}\right)\right], \\
& \mathcal{M}_{i}=\lim _{T \rightarrow \infty} \frac{1}{T} \int_{0}^{T} M_{i} d t=\left(\begin{array}{ll}
m_{i 1} & m_{i 2} \\
m_{i 2} & m_{i 3}
\end{array}\right),
\end{aligned}
$$




$$
\begin{aligned}
& m_{i 1}=\lim _{T \rightarrow \infty} \frac{1}{T} \int_{0}^{T} \cos ^{2} \theta_{i}(t) d t \\
& m_{i 2}=\lim _{T \rightarrow \infty} \frac{1}{T} \int_{0}^{T} \cos \theta_{i}(t) \sin \theta_{i}(t) d t \\
& m_{i 3}=\lim _{T \rightarrow \infty} \frac{1}{T} \int_{0}^{T} \sin ^{2} \theta_{i}(t) d t \\
& { }^{t}\left(X_{i}(0), Y_{i}(0)\right)={ }^{t}\left(x_{i}(0), y_{i}(0)\right)
\end{aligned}
$$

where ${ }^{t}\left(X_{i}, Y_{i}\right)$ is an approximation of ${ }^{t}\left(x_{i}, y_{i}\right)$. Approximation errors in averaging theory are generally bounded only in finite time. However, if an averaged system (which is Eq.(15) here) has an attractor and an original system (which is Eq.(14) here) equilibrates on this attractor, both ${ }^{t}\left(X_{i}, Y_{i}\right)$ and ${ }^{t}\left(x_{i}, y_{i}\right)$ converge to the attractor and an error between them is bounded in $t \in[0, \infty)[10]$. This deduces the fact that if the averaged system has an attractor where the original system (this robotic system) equilibrates and where it has a desired formation, a group formation certainly converges to the desired formation. Therefore, we can examine whether or not the robots form a desired formation by analyzing the averaged system.

Similarly in the case of Eq.(14), the right hand side of Eq.(15) is a linear transformation of a vector field by a matrix, $\mathcal{M}_{i}$. As we have described, the robots stop without forming a desired formation at least when they do not rotate, because $M_{i}$ in Eq.(14) is not a full-rank matrix. This must also hold in Eq.(15), since it is an approximation of Eq.(14). Therefore, we examine the rank of $\mathcal{M}_{i}$ in the following. Obviously, the trace of $\mathcal{M}_{i}$ is positive independently of $\theta_{i}(t)$, i.e., independently of the rotation of the robot. The determinant of $\mathcal{M}_{i}$ is given as:

$$
\begin{array}{r}
\operatorname{det} \mathcal{M}_{i}=\left(\frac{1}{T} \int_{0}^{T} \cos ^{2} \theta_{i}(t) d t\right)\left(\frac{1}{T} \int_{0}^{T} \sin ^{2} \theta_{i}(t) d t\right) \\
-\left(\frac{1}{T} \int_{0}^{T} \cos \theta_{i}(t) \sin \theta_{i}(t) d t\right)^{2}
\end{array}
$$

$T \rightarrow \infty$.

Using Hölder's inequality in functional analysis [11], we can prove $\operatorname{det} \mathcal{M}_{i} \geq 0$. Moreover, we can prove that if and only if the robot does not rotate there exists a constant $c$ such that $\cos \theta_{i}(t)=c \sin \theta_{i}(t)$ and $\operatorname{det} \mathcal{M}_{i}=0$ holds as $M_{i}$. However, if the robot rotates, $\operatorname{det} \mathcal{M}_{i}>0$ holds and $\mathcal{M}_{i}$ is a full-rank matrix whose eigenvalues are positive and then it follows that the robots form a desired formation, (see Subsection 4.2). In other words, the averaged system explicitly describes both of two cases where the non-rotating robots cannot form a desired formation and where the rotating robots can do so, while the original system (which is Eq.(14)) explicitly describes only the former case. Hence, we conclude that we can see stability of this type of nonholonomic mechanical system, which consists of Hilare-type mobile robots, in its averaged system. We particularly emphasize that $\operatorname{det} \mathcal{M}_{i} \geq 0$ is "the most important mathematical formula" in this mechanical system, because this formula gives us a clue to examine stability that we cannot see in the original system. In the following, we give a convincing proof that the averaged system is stable as the desired system.

Let us rewrite the averaged system that is Eq.(15) as Eq.(16):

$\left(\begin{array}{c}\dot{\boldsymbol{X}} \\ \dot{\boldsymbol{Y}}\end{array}\right)=D\left\{\left(\begin{array}{cc}B & 0 \\ 0 & B\end{array}\right)\left(\begin{array}{c}\boldsymbol{X} \\ \boldsymbol{Y}\end{array}\right)+\left(\begin{array}{l}\boldsymbol{d}_{\boldsymbol{x}} \\ \boldsymbol{d}_{\boldsymbol{y}}\end{array}\right)\right\}$

$D=\left(\begin{array}{ll}D_{1} & D_{2} \\ D_{2} & D_{3}\end{array}\right)$

$D_{1}=\operatorname{diag}\left(m_{11}, m_{21}, \cdots, m_{n 1}\right)$,

$D_{2}=\operatorname{diag}\left(m_{12}, m_{22}, \cdots, m_{n 2}\right)$,

$D_{3}=\operatorname{diag}\left(m_{13}, m_{23}, \cdots, m_{n 3}\right)$,

where $\boldsymbol{X}={ }^{t}\left(X_{1}, X_{2}, \cdots, X_{n}\right) ; \boldsymbol{Y}={ }^{t}\left(Y_{1}, Y_{2}, \cdots, Y_{n}\right) ; D \in$ $\mathbf{R}^{2 n \times 2 n} ;$ and $D_{1}, D_{2}, D_{3} \in \mathbf{R}^{n \times n}$. We can see that $D$ is a symmetrical matrix and its all the eigenvalues are real. Since Eq.(16) is identically the same as Eq.(4) that is the desired system except a linear transformation by $D$, Eq.(16) is stable as Eq.(4) if all the eigenvalues of $D$ are positive. Therefore, we examine them in the following. The characteristic equation of $D$ is given as:

$f_{D}(s)=\left|s I_{2 n}-D\right|$,

where $s$ is a complex number; and $I_{2 n}$ is a $2 n \times 2 n$ unit matrix. There exists a matrix, $Q$, that transforms $D$ as:

$Q^{-1} D Q=\left(\begin{array}{cccc}\mathcal{M}_{1} & & & 0 \\ & \mathcal{M}_{2} & & \\ & & \ddots & \\ 0 & & & \mathcal{M}_{n}\end{array}\right)$.

Then, the characteristic equation is rewritten as:

$f_{D}(s)=\prod_{i=1}^{n}\left|s I_{2}-\mathcal{M}_{i}\right|$,

where $I_{2}$ is a $2 \times 2$ unit matrix. As this characteristic equation shows, the eigenvalues of $D$ are the eigenvalues of the $\mathcal{M}_{i}$ 's. Therefore, when all the robots rotate, all the eigenvalues of the $\mathcal{M}_{i}$ 's are positive, i.e., all the eigenvalues of $D$ are positive, and the averaged system is stable certainly as the desired system. Especially, we can choose any non-constant function as $\theta_{i}(t)$ that makes the eigenvalues of $\mathcal{M}_{i}$ positive, i.e., we can design $\theta_{i}(t)$, according to each specific application of this control method. 


\section{Formation Control}

\subsection{Group Formation}

We give a description manner of group formations in this subsection. To describe formations, we define (n-1) relative position vectors between (n) robots as Eq.(17):

${ }^{t}\left(\varphi_{x k}, \varphi_{y k}\right)={ }^{t}\left(x_{j}-x_{i}, y_{j}-y_{i}\right)$,

where $k=1,2, \cdots, n-1$. These vectors are required to form a connected oriented-graph (in which a robot is a node and a relative position vector is an oriented path between robots and in which all the $(n)$ nodes are connected together by the (n-1) oriented paths), otherwise they cannot describe formations uniquely. To separately handle all the $\mathrm{x}$-components and all the $\mathrm{y}$-components of the relative position vectors, we define two vectors as:

$\varphi_{x}={ }^{t}\left(\varphi_{x 1}, \varphi_{x 2}, \cdots, \varphi_{x n-1}\right)$,

$\varphi_{y}={ }^{t}\left(\varphi_{y 1}, \varphi_{y 2}, \cdots, \varphi_{y n-1}\right)$.

The vectors, $\varphi_{x}$ and $\varphi_{y}$, describe the formation along the $x$-axis and along the $y$-axis, respectively, but they do not describe the position of the group, i.e., $\boldsymbol{\varphi}_{\mathfrak{x}}$ and $\varphi_{\boldsymbol{y}}$ do not uniquely correspond to $\boldsymbol{x}$ and $\boldsymbol{y}$. Therefore, we also define two other vectors that include $\varphi_{x}$ and $\varphi_{y}$ as:

$\psi_{x}={ }^{t}\left(\varphi_{x 1}, \varphi_{x 2}, \cdots, \varphi_{x n-1}, \hat{\varphi}_{x}\right)={ }^{t}\left({ }^{t} \varphi_{x}, \hat{\varphi}_{x}\right)$,

$\psi_{y}={ }^{t}\left(\varphi_{y 1}, \varphi_{y 2}, \cdots, \varphi_{y n-1}, \hat{\varphi}_{y}\right)={ }^{t}\left({ }^{t} \varphi_{y}, \hat{\varphi}_{y}\right)$

where $\hat{\varphi}_{x}=x_{1}+x_{2}+\cdots+x_{n}$; and $\hat{\varphi}_{y}=y_{1}+y_{2}+\cdots+y_{n}$. The scalars, $\hat{\varphi}_{x} / n$ and $\hat{\varphi}_{y} / n$, express the position of the center of mass among the robots along the $\mathrm{x}$-axis and along the y-axis, respectively. In other words, $\boldsymbol{\psi}_{\boldsymbol{x}}$ and $\psi_{\boldsymbol{y}}$ express not only the formation but also the position of the group. Therefore, the vectors, $\psi_{\mathfrak{x}}$ and $\psi_{\boldsymbol{y}}$, are uniquely related with $\boldsymbol{x}$ and $\boldsymbol{y}$ as Eq.(18):

$\left(\begin{array}{l}\boldsymbol{\psi}_{\boldsymbol{x}} \\ \boldsymbol{\psi}_{\boldsymbol{y}}\end{array}\right)=F\left(\begin{array}{l}\boldsymbol{x} \\ \boldsymbol{y}\end{array}\right)=\left(\begin{array}{cc}\hat{F} & 0 \\ 0 & \hat{F}\end{array}\right)\left(\begin{array}{l}\boldsymbol{x} \\ \boldsymbol{y}\end{array}\right)$

$\hat{F}=\left(\begin{array}{c}f_{1} \\ f_{2} \\ \vdots \\ f_{n}\end{array}\right)$

$\boldsymbol{f}_{i}=(\cdots, 1, \cdots,-1, \cdots), i<n$,

$f_{n}=(1,1, \cdots, 1)$,

where $F \in \mathbf{R}^{2 n \times 2 n} ; \hat{F} \in \mathbf{R}^{n \times n} ;$ and $f_{i} \in \mathbf{R}^{1 \times n}$. All the components of $f_{i}, i<n$, are zero except two of them: 1 and -1 . The $f_{i}$ 's are linearly independent of each other, which means that the relative position vectors form a connected oriented-graph mentioned above.

\subsection{Formation Controllability}

In this subsection, we prove that formation controllability holds when the robots rotate. Specifically; we first show that formation controllability holds in the desired system in which the robots are assumed to be holonomic, and we next show that this controllability also holds both in the averaged system and in the original system, when the robots that are nonholonomic rotate. Particularly, since, as we prove below, this controllability is common in these systems, the desired, averaged and original systems, we can estimate the final formation certainly in the desired system that is just a first order linear time differential equation and that is independent of the rotations of the robots.

Using Eq.(18), the desired system that is Eq.(4) is rewritten as Eq.(19):

$$
\left(\begin{array}{c}
\dot{\psi}_{x} \\
\dot{\psi}_{y}
\end{array}\right)=\left(\begin{array}{cc}
\hat{F} B \hat{F}^{-1} & 0 \\
0 & \hat{F} B \hat{F}^{-1}
\end{array}\right)\left(\begin{array}{l}
\psi_{x} \\
\psi_{y}
\end{array}\right)+\left(\begin{array}{l}
\hat{F} d_{x} \\
\hat{F} d_{y}
\end{array}\right)
$$

Since the n-th column vector of $\hat{F}^{-1}$ is a kernel of $B$, the n-th column vector of $\hat{F} B \hat{F}^{-1}$ is a zero vector as Eq. (20):

$\hat{F} B \hat{F}^{-1}=\left(\begin{array}{cc} & 0 \\ A & \vdots \\ & 0 \\ a & 0\end{array}\right)$,

where $A \in \mathbf{R}^{(n-1) \times(n-1)}$; and $\boldsymbol{a} \in \mathbf{R}^{1 \times(n-1)}$. This matrix physically means that $\varphi_{x}$ and $\varphi_{y}$ are independent of $\hat{\varphi}_{x}$ and $\hat{\varphi}_{y}$, i.e., the formation is independent of the position of the group. The final location of the group depends on its initial location as $B$ has a kernel, i.e., a zero-eigenvalue that is stable but not asymptotically stable. Consequently, from Eq.(19), we can extract Eq.(21):

$$
\left(\begin{array}{l}
\dot{\varphi}_{x} \\
\dot{\varphi}_{y}
\end{array}\right)=\left(\begin{array}{cc}
A & 0 \\
0 & A
\end{array}\right)\left(\begin{array}{l}
\varphi_{x} \\
\varphi_{y}
\end{array}\right)+\left(\begin{array}{l}
\hat{P} d_{x} \\
\hat{P} d_{y}
\end{array}\right)
$$

where $\hat{P} \in \mathbf{R}^{(n-1) \times n}$. The matrix, $\hat{P}$, is derived by eliminating the $\mathrm{n}$-th row vector of $\hat{F}$. For formation controllability to hold, $A$ must be asymptotically stable and $P$ must be full-rank. Therefore, we examine stability of $A$ here. As Eq.(20) shows, $A$ is derived from $B$ by a linear transformation, so that the characteristic equation of $B$ is modified as Eq.(22):

$f_{B}(s)=\left|s I_{n}-B\right|=s\left|s I_{n-1}-A\right|=s f_{A}(s)$,

where $s$ is a complex number; $I_{n}$ is a $n \times n$ unit matrix; and $I_{n-1}$ is a $(n-1) \times(n-1)$ unit matrix. Since, as Eq.(22) shows, all the eigenvalues of $B$ except a zero-eigenvalue are identically the same as those of $A$ and, as we have described in Subsection 3.2, they are asymptotically stable, we can see that $A$ is asymptotically 
stable. Obviously, $\hat{P}$ is full-rank. Hence, we conclude that $\varphi_{x}$ and $\varphi_{y}$ are controllable by $d_{x}$ and $d_{y}$, respectively, i.e., the formation is controllable, in the desired system. We can, of course, satisfy the condition for stability: $d_{x}$, $\boldsymbol{d}_{\boldsymbol{y}} \in S$ that has been described in Subsection 3.2 on controlling the format:on, because the condition restricts just one degree of freedom along each of the $\mathrm{x}$ - and $\mathrm{y}$-axes and we have the $(\mathrm{n})$-dimensional control inputs, $\boldsymbol{d}_{\boldsymbol{x}}$ and $\boldsymbol{d}_{\boldsymbol{y}}$, for the (n-1)-dimensional controlled states, $\boldsymbol{\varphi}_{\boldsymbol{x}}$ and $\boldsymbol{\varphi}_{\boldsymbol{y}}$.

Similarly in the case of Eq.(18), we define a vector as:

$$
\left(\begin{array}{l}
\boldsymbol{\Psi}_{\boldsymbol{x}} \\
\boldsymbol{\Psi}_{\boldsymbol{y}}
\end{array}\right)=F\left(\begin{array}{l}
\boldsymbol{X} \\
\boldsymbol{Y}
\end{array}\right)=\left(\begin{array}{cc}
\hat{F} & 0 \\
0 & \hat{F}
\end{array}\right)\left(\begin{array}{l}
\boldsymbol{X} \\
\boldsymbol{Y}
\end{array}\right)
$$

where

$$
\begin{aligned}
& \boldsymbol{\Psi}_{\boldsymbol{x}}={ }^{t}\left(\Phi_{x 1}, \Phi_{x 2}, \cdots, \Phi_{x n-1}, \hat{\Phi}_{x}\right)={ }^{t}\left({ }^{t} \boldsymbol{\Phi}_{x}, \hat{\Phi}_{x}\right), \\
& \boldsymbol{\Psi}_{\boldsymbol{y}}={ }^{t}\left(\Phi_{y 1}, \Phi_{y 2}, \cdots, \Phi_{y n-1}, \hat{\Phi}_{y}\right)={ }^{t}\left({ }^{t} \boldsymbol{\Phi}_{\boldsymbol{y}}, \hat{\Phi}_{y}\right),
\end{aligned}
$$

and then we rewrite the averaged system that is Eq.(16) as Eq. (23):

$$
\begin{array}{r}
\left(\begin{array}{c}
\dot{\mathbf{\Psi}}_{\boldsymbol{x}} \\
\dot{\mathbf{\Psi}}_{\boldsymbol{y}}
\end{array}\right)=F D F^{-1}\left\{\left(\begin{array}{cc}
\hat{F} B \hat{F}^{-1} & 0 \\
0 & \hat{F} B \hat{F}^{-1}
\end{array}\right)\left(\begin{array}{l}
\boldsymbol{\Psi}_{\boldsymbol{x}} \\
\boldsymbol{\Psi}_{\boldsymbol{y}}
\end{array}\right)\right. \\
\left.+\left(\begin{array}{l}
\hat{F} d_{\mathfrak{x}} \\
\hat{F} d_{\boldsymbol{y}}
\end{array}\right)\right\}
\end{array}
$$

where $\boldsymbol{\Psi}_{\boldsymbol{x}}$ and $\boldsymbol{\Psi}_{\boldsymbol{y}}$ are approximations of $\boldsymbol{\psi}_{\boldsymbol{x}}$ and $\boldsymbol{\psi}_{\boldsymbol{y}}$, respectively. The vector field in the braces of Eq.(23) is identically the same as the vector field in the right hand side of Eq. (19) and this vector field is transformed by $F D F^{-1}$. Since, as we have proven, all the eigenvalues of $D$ are positive when the robots rotate, the averaged system in Eq.(23) is stable as the desired system in Eq.(19). Specifically, as Eq.(21) extracted from Eq.(19) shows, $\varphi_{x}$ and $\varphi_{y}$ in the desired system converge to an attractor where the group has a formation determined by $d_{x}$ and $d_{y}$, so that $\boldsymbol{\Phi}_{\boldsymbol{x}}$ and $\boldsymbol{\Phi}_{\boldsymbol{y}}$ in the averaged system also converge to the same attractor. Of course, the original system equilibrates on this attractor, which deduces the fact that the attractor is also an attractor in the original system, i.e., the robots, which are Hilare-type mobile robots, form a formation which is exactly the same as that determined by Eq.(21). Hence, formation controllability holds and it is common in the desired, averaged and original systems.

\section{Simulations}

We simulated to form V-Formation which is a character "V" rotated by $-\pi / 2.0$ [rad]. In these simulations, the robot group consists of eight Hilare-type mobile robots and it has an open chain group configuration. They are initially placed on the line, $y=3.0$, at intervals of $0.5[\mathrm{~m}]$ and their initial orientations are $\theta_{i}(0)=\pi / 2.0[\mathrm{rad}], i=1,2, \cdots, 8$, (see Figure 3 ). The radius of the robot wheel and the width between the wheels are given as: $R=0.05[\mathrm{~m}]$ and $W=0.15[\mathrm{~m}]$. The attraction coefficients are set as: $\tau_{i j}=\tau_{j i}=4.0, j=i+1$, $i=1,2, \cdots, 7$. To form this formation, we set the formation vectors as: ${ }^{t}\left(d_{x 1}, d_{y 1}\right)={ }^{t}(2.0,-2.0) ;{ }^{t}\left(d_{x 4}, d_{y 4}\right)$ $={ }^{t}\left(d_{x 5}, d_{y 5}\right)={ }^{t}(-2.0,0.0) ;{ }^{t}\left(d_{x 8}, d_{y 8}\right)={ }^{t}(2.0,2.0)$; and ${ }^{t}\left(d_{x i}, d_{y i}\right)={ }^{t}(0.0,0.0), i=2,3,6,7$. Since these formation vectors satisfy the condition for stability: $d_{x}, d_{y} \in S$ that has been described in Subsection 3.2, the robot group does not drift.

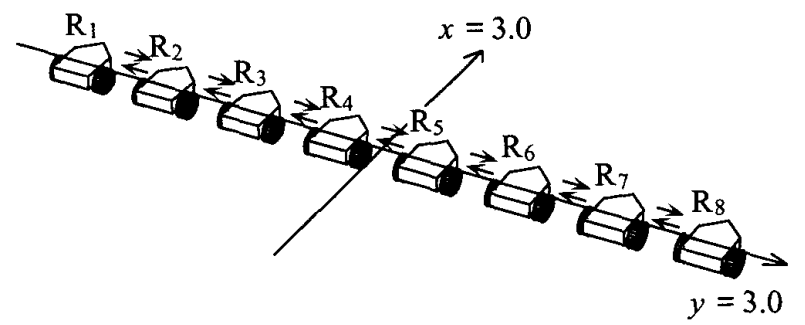

Figure 3 An Open Chain Group

Figure 4 shows a result where the robot rotates as: $\dot{\theta}_{i}(t)$ $=\omega_{i}=2.0 \pi / 10.0[\mathrm{rad} / \mathrm{sec}]$. We can see that the robots form V-Formation. Since the robot still continues to rotate after forming the formation, the locus of the robot motion makes a circle around its final location. This may be undesirable in many applications. The following example shows a more practically useful approach in which the robot stops rotating after forming the formation.

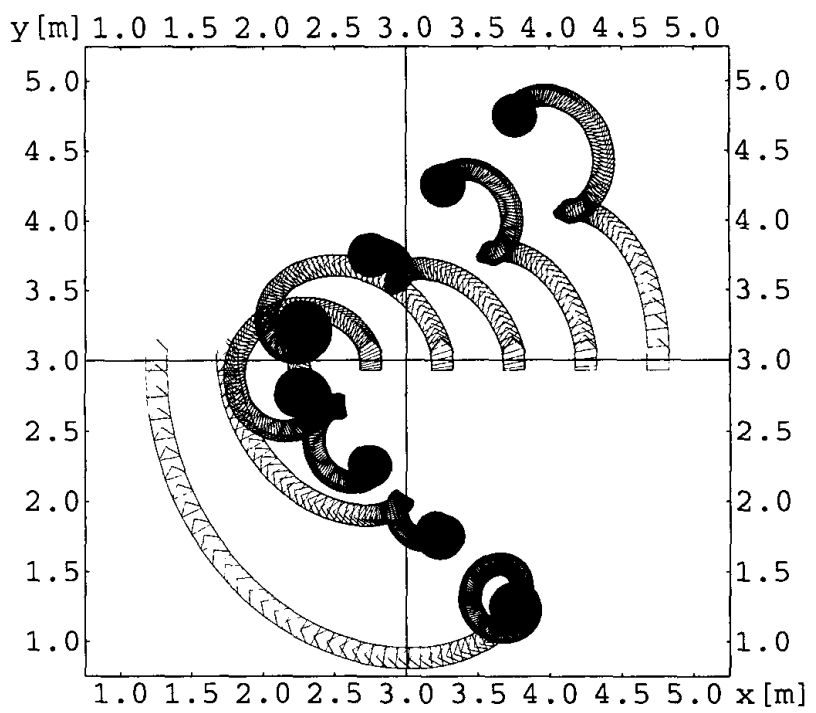

Figure 4 A Simulation Result

Figure 5 shows a result where the robot rotates (oscillates) as:

$\dot{\theta}_{i}(t)=\tau\left\{\left(\theta_{0}+A_{i}\left(\tilde{v}_{i}\right) \sin \omega_{i} t\right)-\theta_{i}(t)\right\}$, 
$A_{i}\left(\tilde{\boldsymbol{v}}_{\boldsymbol{i}}\right)=\left\{\begin{array}{cc}A_{0} & k\left\|\tilde{\boldsymbol{v}}_{\boldsymbol{i}}\right\|>A_{0} \\ k\left\|\tilde{\boldsymbol{v}}_{\boldsymbol{i}}\right\| & k\left\|\tilde{\boldsymbol{v}}_{\boldsymbol{i}}\right\| \leq A_{0}\end{array}\right.$

where $\tau=4.0 ; \theta_{0}=\pi / 2.0[\mathrm{rad}] ; \omega_{i}=2.0 \pi / 5.0[\mathrm{rad} / \mathrm{sec}]$; $k=50.0 ;$ and $A_{0}=\pi / 8.0[\mathrm{rad}]$. As the above equation shows, as long as $\left\|\tilde{\boldsymbol{v}}_{i}\right\|$ is larger than $A_{0} / k(=\pi / 400.0)$, the perturbation amplitude, $A_{i}\left(\tilde{\boldsymbol{v}}_{\boldsymbol{i}}\right)$, is $\pi / 8.0[\mathrm{rad}]$, so that the robot rotates (oscillates). However, as $\left\|\tilde{\boldsymbol{v}}_{\boldsymbol{i}}\right\|$ converges to zero, i.e., as the formation converges to the final formation, the amplitude also converges to zero and the robot finally stops rotating. Its final orientation is $\theta_{0}(=$ $\pi / 2.0[\mathrm{rad}])$. Hence, the robot can control its final position to form a desired formation and it can also control its final orientation.

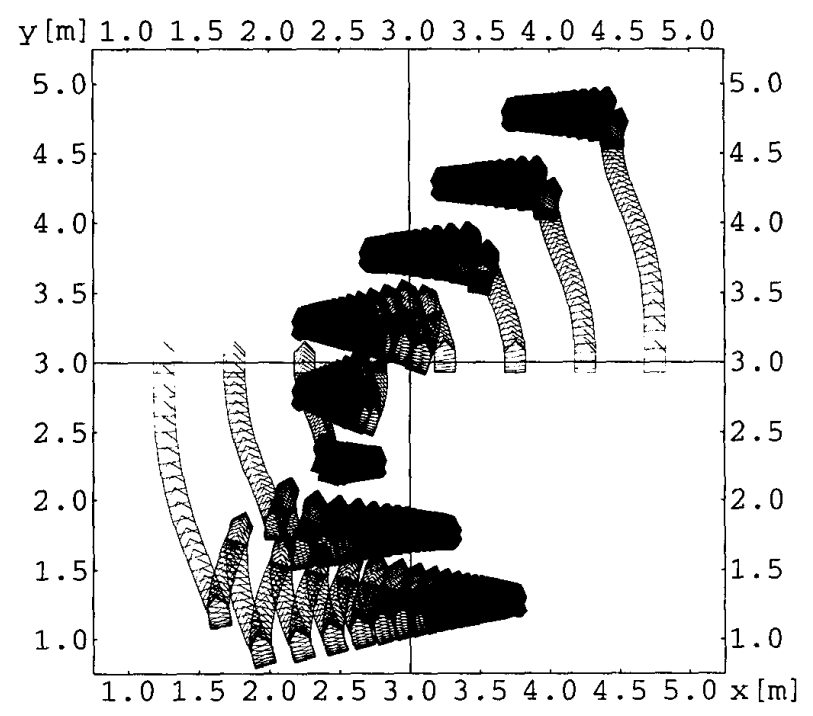

Figure 5 A Simulation Result

The rotating motions of all the robots are synchronized in these simulations. Of course, the robots form the same final formation in asynchronous cases, because the final formation is independent of the rotating motions. It is determined solely by the formation vectors and it is exactly described by Eq.(21). Hence, the validity of this control method is verified in these simulations.

Collision avoidance strategy between robots (e.g., potential field methods) can be easily implemented in the desired system and asymptotic stability of this case can be shown identically.

\section{Conclusions}

We showed potentiality of using averaging theory to examine stability of feedback control in nonholonomic mechanics and we proved that asymptotic stability is guaranteed by a smooth time-varying feedback control law in cases of multiple Hilare-type mobile robots to form group formations. Particularly, it is advantage that we can choose any non-constant time function as the orientation of the Hilare-type mobile robot according to each specific application. Hence, this method allows for a wide variety of time-varying feedback. There are important problems unsolved: (i) to show the convergence rate of this control method; and (ii) to apply this control method to other mechanical systems, e.g., automobiles. We will investigate these problems in future.

\section{References}

[1] R. W. Brockett, "Asymptotic stability and feedback stabilization," R. W. Brockett, R. S. Millman and H. J. Sussman, Eds., Differential Geometric Control Theory, Boston: Birkhauser, pp. 181-208, 1983.

[2] C. Samson, "Velocity and torque feedback control of a nonholonomic cart," in Proc. Int. Workshop in Adaptive and Nonlinear Control: Issues in Robotics, Grenoble, France, 1990.

[3] J. M. Coron, "Global asymptotic stabilization for controllable systems without drift," Mathematics of Control, Signals and Systems, vol.5, no.3, pp. 295-312, 1992.

[4] J. B. Pomet, "Explicit design of time-varying stabilizing control laws for a class of controllable systems without drift," Syst. Contr. Lett., vol.18, no.2, pp. 147-58, Feb. 1992.

[5] A. R. Teel, R. M. Murray and G. Walsh, "Non-holonomic control systems: From steering to stabilization with sinusoids," Int. J. Contr., vol. 62, no. 4, pp. 849-870, 1995.

[6] C. Samson, "Control of chained systems: Application to path following and time-varying point-stabilization of mobile robots," IEEE Trans. Automat. Contr., vol. 40, no. 1, pp. 64-77, 1995.

[7] A. M. Bloch, M. Reyhanoglu and N. M. McClamroch, "Control and stabilization of nonholonomic dynamic systems," IEEE Trans. Automat. Contr., vol. 37, no. 11, pp. 1746-1757, 1992.

[8] C. Canudas de Wit and O. J. Sørdalen, "Exponential stabilization of mobile robots with nonholonomic constraints," IEEE Trans. Automat. Contr., vol. 37, no. 11, pp. 1791-1797, 1992.

[9] O. J. Sørdalen and O. Egeland, "Exponential stabilization of nonholonomic chained systems," IEEE Trans. Automat. Contr., vol. 40, no. 1, pp. 35-49, January 1995.

[10] J. A. Sanders and F. Verhulst, "Averaging methods in nonlinear dynamical systems," New York: Springer-Verlag, 1985.

[11] K. Yosida, "Functional analysis," Berlin Heidelberg: Springer-Verlag, 6th Ed., 1980.

[12] S. Kodama and N. Suda, "Matrix theory for system control," the Society of Instrument and Control Engineers (SICE in Japanese), 1978. 